\title{
ON THE ARONSON-JOHNSON-LAMBERT DECOMPOSITION OF THE REDISTRIBUTIVE EFFECT
}

\author{
Achille Vernizzi Simone Pellegrino
}

Working Paper n. 2007-13

GIUGNO 2007

U I I I UNIVERSITẢ DEGLI STUDI DI MILANO

\section{Dipartimento di SCIenze Economiche AzIendali e Statistiche}

Via Conservatorio 7

20122 Milano

tel. ++390250321501 (21522) - fax ++390250321450 (21505)

http://www.economia.unimi.it

E Mail: dipeco@unimi.it 


\title{
ON THE ARONSON-JOHNSON-LAMBERT DECOMPOSITION OF THE REDISTRIBUTIVE EFFECT
}

\author{
Achille Vernizzi • Simone Pellegrino
}

\begin{abstract}
Recently van De Van, Creedy and Lambert (2001) and Lambert and Urban (2005) have reconsidered the original Aronson, Johnson and Lambert (1994) decomposition of the redistributive effect in order to properly evaluate personal income tax reforms, when sequential income groups do not concern exact equals. Lambert and Urban (2005) decompose the Atkinson-Plotnick-Kakwani index into three terms. We utilize this decomposition in choosing the optimal bandwidth set and suggest to consider not only the highest vertical contribution to the redistributive effect, but also the horizontal inequity due to the reranking of the mean post-tax income among groups. Findings are applied to Italian data with respect to both individual nominal incomes and equivalent household incomes.
\end{abstract}

JEL Classification Numbers: H23; H24.

Keywords: Personal Income Tax, Redistributive Effect, Horizontal Inequity, Reranking.

\section{Introduction}

Decomposing redistributive effect across groups of pre-tax equals into vertical, horizontal and reranking effect has been intensively studied in the last years. The original work by Aronson, Johnson and Lambert (1994) considers exact pre-tax equals in portioning the pre-tax income distribution.

As van de Ven, Creedy and Lambert (2001) pointed out, in the real word taxation this is not the case: only groups with close pre-tax incomes can be considered. They got through this problem in order to individuate the optimal bandwidth that should be used in decomposing the redistributive effect as the Aronson, Johnson and Lambert (1994) methodology suggests.

\footnotetext{
- Dipartimento di Scienze Economiche, Aziendali e Statistiche, Università degli Studi di Milano. E-mail: achille.vernizzi@unimi.it. • Dipartimento di Scienze Economiche e Finanziarie "G. Prato", Università degli Studi di Torino. E-mail: spellegrino@gmail.com.
} 
In this work we come back to this point by proposing a new measurement methodology. We focus on the idea that the optimal bandwidth should be individuated by looking not only to the highest vertical contribution to the redistributive effect, but also to the horizontal inequity due to the reranking of the mean post-tax income among groups. In doing so we propose a decomposition of the Atkinson-Plotnick-Kakwani index in three terms, following the decompositions applied in Lambert and Urban (2005). Using this decomposition, the optimal bandwidth can be individuated without leading to misleading results: a bandwidth with which the vertical effect is the highest and there is no reranking of the mean post-tax income among groups can be considered the best one to be adopted.

Findings are applied to Italian data with respect to both individual nominal incomes and equivalent household incomes. Following Ebert and Moyes (2000) household analysis we studied the changes in the redistributive effect decomposition using different equivalent scales and different definitions of income accruing to each individual within the household. Then we analyze the Personal Income Tax reforms proposed in Italy in the period 2004-2007 and give some suggestions on the goodness of these reforms.

The structure of the paper is as follows. In Section 2 we revisit the original Aronson, Johnson and Lambert (1994) decomposition. Section 3 explains the elaborations we propose to individuate the optimal bandwidth. In Section 4 we suggest some useful interpretations for evaluating personal income tax reforms in Italy, decomposing the redistributive effect using a bandwidth which falls within the "optimal" bandwidth set.

\section{Aronson-Johnson-Lambert $R E$ and Atkinson-Plotnick- Kakwani $R$ indexes re-examined}

Let $G_{y}$ and $G_{y-T}$ be the Gini index on the gross and net incomes respectively. The redistributive index $R E$ is equal to $R E=G_{y}-G_{y-T}$. It is well-known that the Gini coefficient fails to decompose across subgroups into between- and within-group 
inequality components in case the subgroup income ranges overlap. Aronson, Johnson and Lambert (1994) consider the following decomposition of the post-tax Gini coefficient: $R E=\left(G_{y}^{B}-G_{y-T}^{B}\right)+\left(G_{y}^{W}-G_{y-T}^{W}\right)+\left[\left(G_{y}-C_{y}\right)-\left(G_{y-T}-C_{y-T}\right)\right]$, where $G_{y}^{B}$ and $G_{y-T}^{B}$ are the between-group Gini coefficients, $G_{y}^{W}$ and $G_{y-T}^{W}$ are the within-group Gini coefficients, $C_{y}$ and $C_{y-T}$ are the post-tax concentration coefficients.

If pre-tax groups are chosen partitioning an income parade in non decreasing order, such that the maximum of a group is not greater than the minimum of the following group, then $G_{y}=C_{y}$. Moreover, even if they do not make an explicit statement, Aronson, Johnson and Lambert (1994) implicitly assume that after taxation (i) the group average incomes maintain the same ranking as before taxation and (ii) the within group orderings remain the same as before taxation. If this is the case $R E=\left(G_{y}^{B}-G_{y-T}^{B}\right)+\left(G_{y}^{W}-G_{y-T}^{W}\right)-R$, being $R$ the Atkinson-Plotnick-Kakwani reranking index.

Aronson, Johnson and Lambert (1994) originally limit the analysis to the case in which the population groups contain the exact pre-tax equals. This implies $G_{y}^{W}=0$, so that $G_{y}^{B}=G_{y}$. In this special case $R E$ can be further simplified and written as $G_{y}-G_{y-T}^{B}-G_{y-T}^{W}-R$, where $G_{y}-G_{y-T}^{B}$ is the vertical potential redistribution effect, which looses part of its potentiality whenever, after taxation, either within group inequality index $G_{y-T}^{W}$ or group overlapping index (equal to the Atkinson-PlotnickKakwani reranking index in this special case) $R=G_{y-T}-C_{y-T}$ becomes different from zero.

However, as observed before, this decomposition can be correctly applied provided that each group is composed by observations with the same pre-tax income and taxation does not modify either the ranking among group averages or the within group rankings (van de Ven, Creedy and Lambert, 2001; Urban and Lambert, 2005; Vernizzi, 2006). In the real word taxation $G_{y}^{W}$ is generally different from zero, as only groups with close pre-tax incomes can be considered. As a consequence, only bandwidths of income containing close-equals must be chosen. 
Being more general, neither the mean post-tax income of each group maintains the same order of the mean pre-tax income of each group nor, within each group, the order of the incomes remains unchanged in the transition from the pre- to the post-tax incomes; then the residual of the $R E$ decomposition is generally not equal to the Atkinson-Plotnick-Kakwani index. We happened to observe these violations using a SHIW dataset, even if the magnitude of these unpleasant outcomes depends on the income range (bandwidth) chosen for each group. In particular the bigger the income range defining each group, the less likely group average incomes overlap in the transition from the pre- to the post-tax incomes; the opposite case happens to within group incomes. The income bandwidth acts in the same direction towards group reranking and within group reranking: the larger the bandwidth is, the less probable is the former and the more frequent happens to be the latter.

In addition, as the bandwidth increases, $G_{y}^{W}$ can be no more close to zero, so that $R E$ cannot be no more evaluated as $G_{y}-G_{y-T}^{B}-G_{y-T}^{W}-R=G_{y}^{B}-G_{y-T}^{B}-G_{y-T}^{W}-R$, rather it becomes more realistic to turn back to the more complete decomposition $R E=\left(G_{y}^{B}-G_{y-T}^{B}\right)+\left(G_{y}^{W}-G_{y-T}^{W}\right)-R^{*}=V-H-R^{*}$, having defined $H=G_{y-T}^{W}-G_{y}^{W}$ and $R^{*}=G_{y-T}-C_{y-T}^{*}$, being $C_{y-T}^{*}$ the concentration index for the after tax income parade ordered (i) according to the after tax ranking for group income average and (ii) such that within group incomes are in not decreasing order ${ }^{1}$.

The decomposition proposed in van de Ven, Creedy and Lambert (2001) shows that an arbitrary specification of close-equals groups can lead to misleading results; it cannot consider all the inequality-related elements in the subgroup analysis.

In this work we give back the idea of constituting close-equals groups, and focus on the eventual enlargement of the within group inequality $\left(G_{y-T}^{W}-G_{y}^{W}\right)=H$ term, together with the group overlapping term $R^{*}$, to measure the loss in potential vertical redistribution effect measured by $\left(G_{y}^{B}-G_{y-T}^{B}\right)=V$.

\footnotetext{
${ }^{1}$ We observe that generally $G_{y}^{B} \geq G_{y-T}^{B}$, whilst $G_{y}^{W} \leq G_{y-T}^{W}$, so that $\left(G_{y}^{B}-G_{y-T}^{B}\right)=V \geq 0$ and $\left(G_{y}^{W}-G_{y-T}^{W}\right)=-H \leq 0$.
} 
As Urban and Lambert (2005) show ${ }^{2}$, the difference between $\left(G_{y-T}-C_{y-T}\right)$ is not necessarily limited to the overlapping or transvariation $\operatorname{term}^{3} R^{*}$; it may include also a reranking effect for group averages together with a within group reranking effect: moreover it is likely that the former can vanish only if the latter increases, thanks to an enlargement of the bandwidth.

The Atkinson-Plotnick-Kakwani index can then be decomposed into three terms: $R=G_{y-T}-C_{y-T}=\left(G_{y-T}-C_{y-T}^{*}\right)+\left(C_{y-T}^{*}-C_{y-T}^{\circ}\right)+\left(C_{y-T}^{\circ}-C_{y-T}\right) \quad$ where $C_{y-T}^{\circ}$ is the concentration index of the post-tax incomes provided that post-tax incomes maintain the same order within each group (condition (ii)) without imposing condition (i), i.e. the post-tax group average incomes are in non decreasing order. The above specified components of the Atkinson-Plotnick-Kakwani index may be defined as:

- $R_{1}=G_{y-T}-C_{y-T}^{*}=R^{*}$ is the transvariation index among groups;

- $R_{2}=C_{y-T}^{*}-C_{y-T}^{\circ}$ measures the horizontal inequity due to the reranking of the mean post-tax income among groups;

- $R_{3}=C_{y-T}^{\circ}-C_{y-T}$ measures the horizontal inequity due to the reranking within groups.

In so doing we obtain $R=R_{1}+R_{2}+R_{3}$, where $R_{1}=R^{*}$. So, thanks to $R E$ we can still evaluate how horizontal inequity affects vertical redistribution potentiality, being horizontal inequity measured by $H=\left(G_{y}^{W}-G_{y-T}^{W}\right)$ and by $R^{*}=G_{y-T}-C_{y-T}^{*}$; thanks to the decomposition of $R$ we can further control for income average reranking among groups and measure within group reranking of incomes after taxation. In our opinion what should be avoided is the income average reranking and, in the meanwhile, as van de Ven, Creedy and Lambert (2001) suggest, $\frac{V}{R E}$ should be maximized.

\footnotetext{
${ }^{2}$ See also Vernizzi (2006) for analytical details.

${ }^{3}$ Transvariation is a term used by Dagum (1997).
} 


\section{On determining the optimal bandwidth}

This section explains how the $R E$ decomposition and the extension of the AtkinsonPlotnick-Kakwani index can be useful to evaluate the effects of changes in the Personal Income Tax. We analyze the redistributive effect of the Italian Personal Income Tax during the 2004 to 2007 year with respect both to individuals and to households ${ }^{4}$.

In order to convert households' incomes into equivalent incomes we adopt the Cutler scale. If we consider the $h$-th family which has $N_{h}$ components split into $N A_{h}$ adults and $N C_{h}$ children aged 18 or less, the Cutler scale is given by the expression $C S_{h}=\left(N A_{h}+\alpha N C_{h}\right)^{\beta}$, where $\alpha$ and $\beta$ are two parameters that range between zero and one. In this section the decomposition has been investigated for $\alpha=0.5$ and $\beta=0.65$. Ebert and Moyes (2000) observe that, in applying equivalence scales, the choice of the weight may be arbitrary: we consequently decided to weigh equivalent incomes either by the lower and the upper bound, the former being $1^{5}$ and the latter being the component number associated to each family ${ }^{6}$. We label the equivalent income parade $m(1)$ if the weight attributed to each family is 1 , and we label the same income parade as $m(n)$, if the weight is equal to the number of family components.

Figures 1-9 show the percentage measures of $\frac{V}{R E}-100, \frac{H}{R E}, \frac{R^{*}}{R E}$, and the percentage of $R_{1}, R_{2}$ and $R_{3}$ on $R$ calculated using bandwidths $w$ that range between 10 to 3,000 euro. The graphs for the $R E$ and $R$ components show a quite similar behavior either for individual incomes or equivalent incomes in each of the three years here considered. $\frac{V}{R E}$ initially increases up to bandwidths around 300 euro, then is constant and, finally, shows a decreasing trend. The line becomes the more irregular the more it departs from the axes origin: the irregularities are more similar to irregular waves than to completely random white noises.

\footnotetext{
${ }^{4}$ The 2004 Italian SHIW dataset provides demographic and post-tax income microdata for a representative cross-section of 12,713 taxpayers and 8,012 households (20,581 individuals). This data were used to obtain gross and net incomes according to the Italian Personal Income Tax (Pellegrino, 2007b). Once the 2004 gross income parade was obtained, the 2006 and 2007 distribution was obtained considering the impact of the inflation rate (Pellegrino, 2007c).

${ }^{5} \alpha=\beta=0$.

${ }^{6} \alpha=\beta=1$.
} 
Figure 1: $V / R E, H / R E, R * / R E$ and $R \%$ decomposition - Individuals in 2004

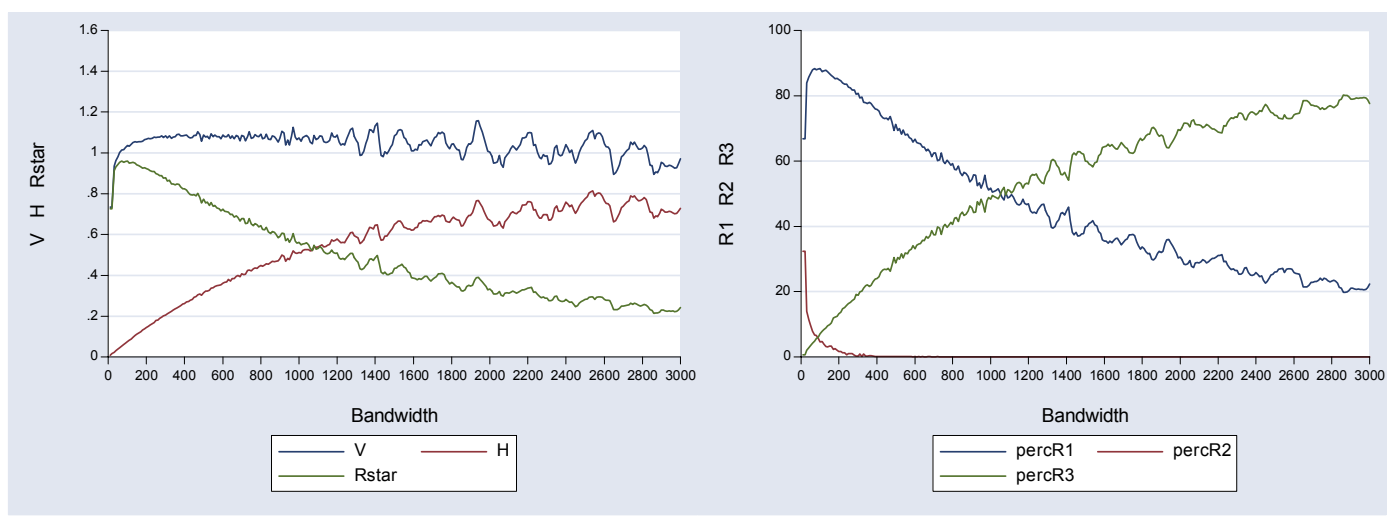

Figure 2: $V / R E, H / R E, R * / R E$ and $R \%$ decomposition - Individuals in 2006

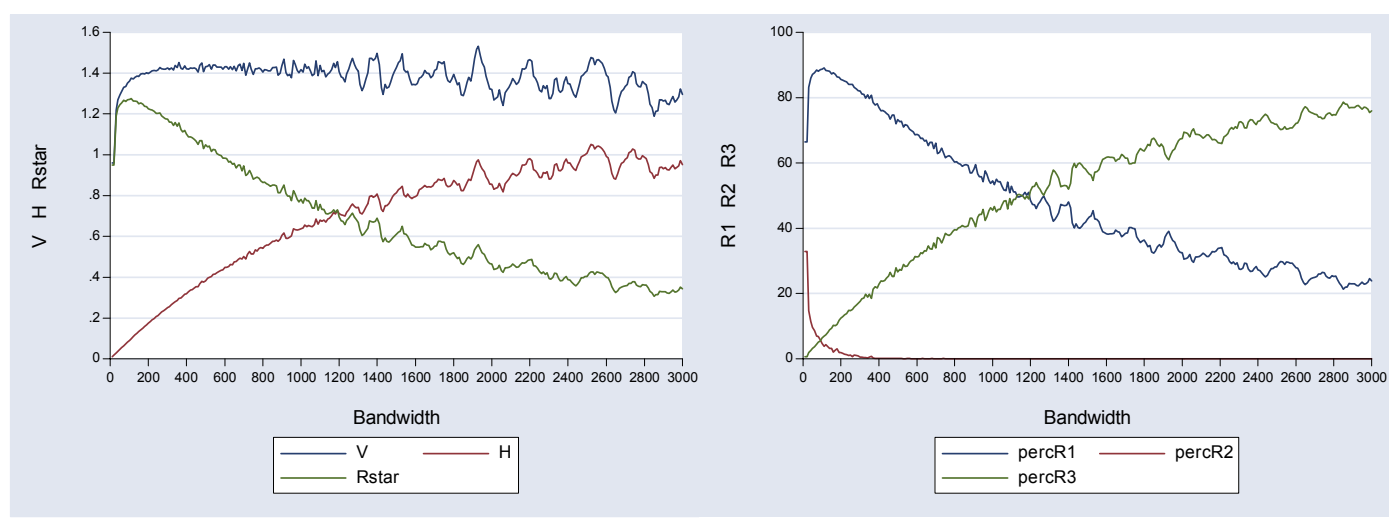

Figure 3: V/RE, $H / R E, R * / R E$ and $R \%$ decomposition - Individuals in 2007

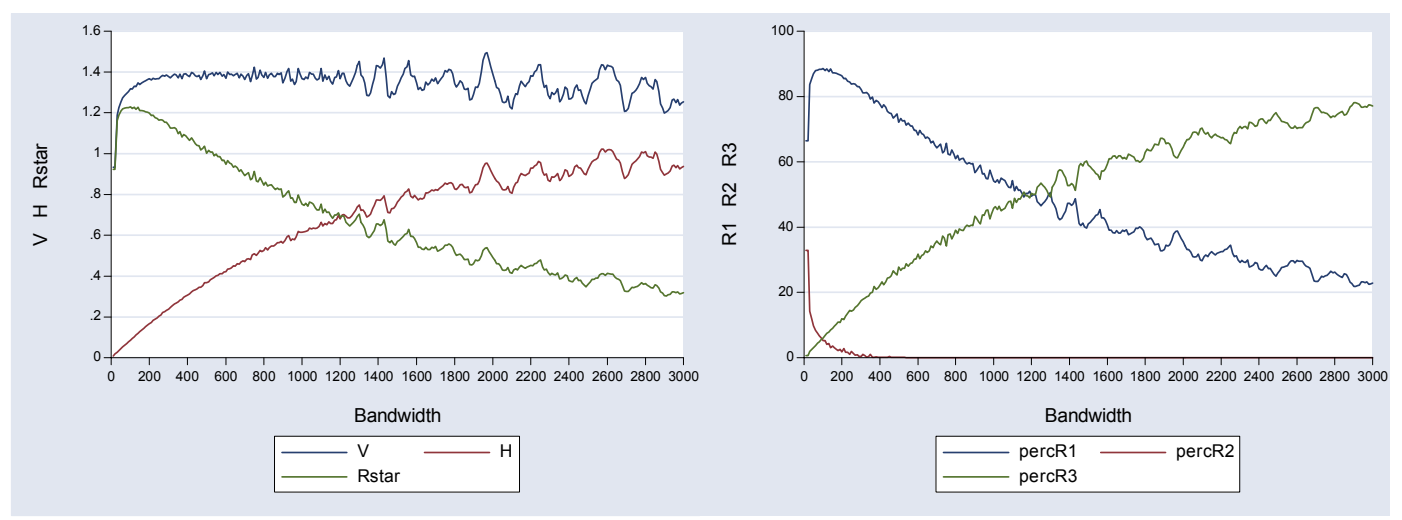


Figure 4: V/RE, H/RE, $R * / R E$ and $R \%$ decomposition $-m(1)$ Households in 2004 §
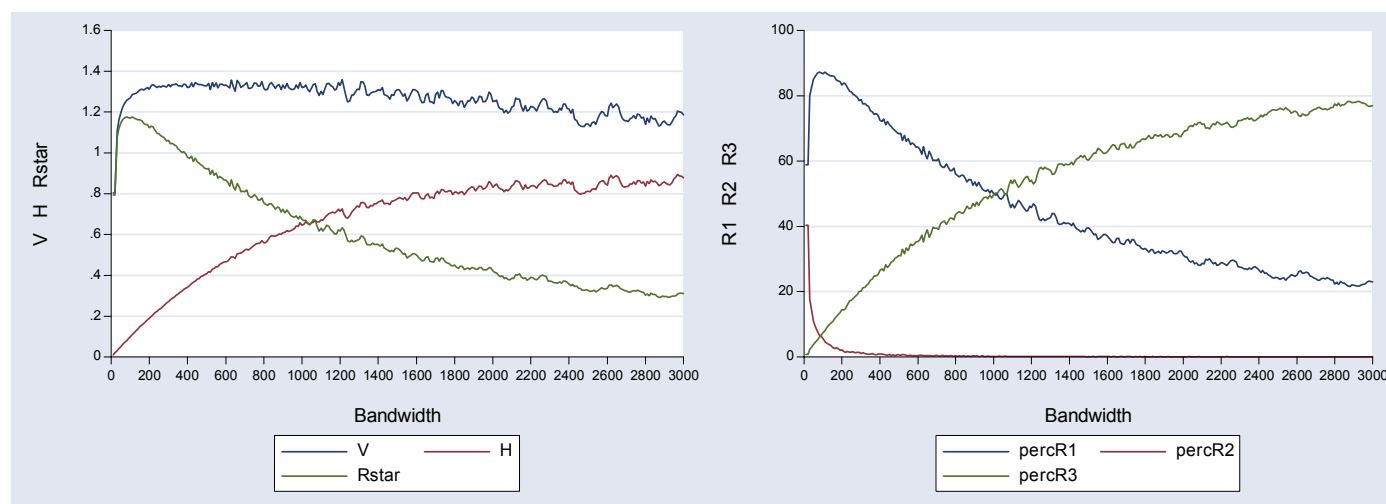

Figure 5: V/RE, $H / R E, R * / R E$ and $R \%$ decomposition $-m(1)$ Households in $2006^{\S}$
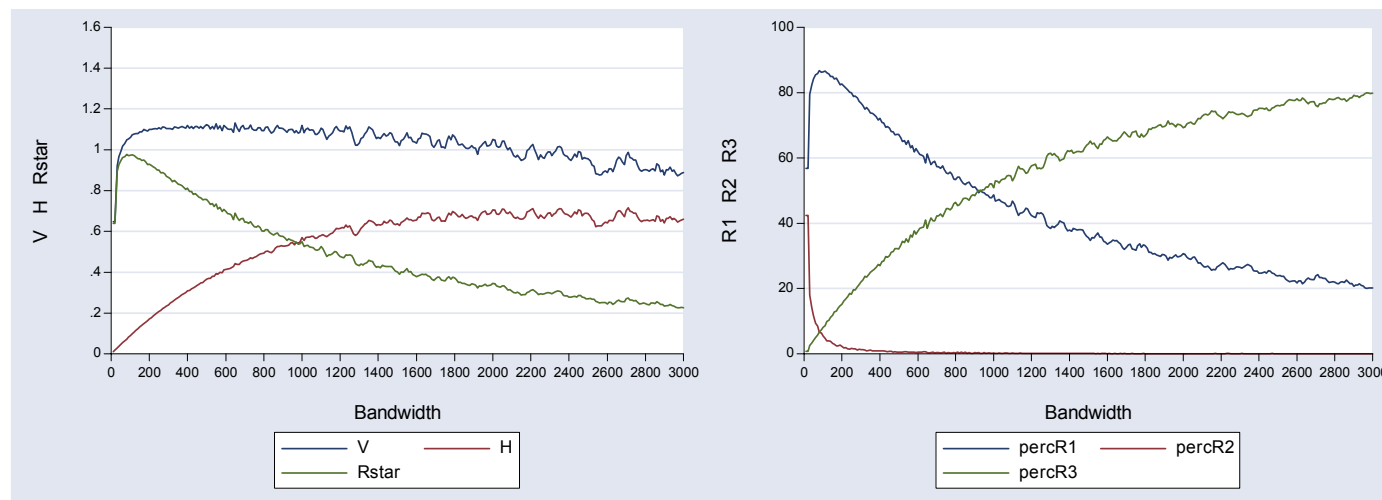

Figure 6: $V / R E, H / R E, R * / R E$ and $R \%$ decomposition $-m(1)$ Households in 2007 §
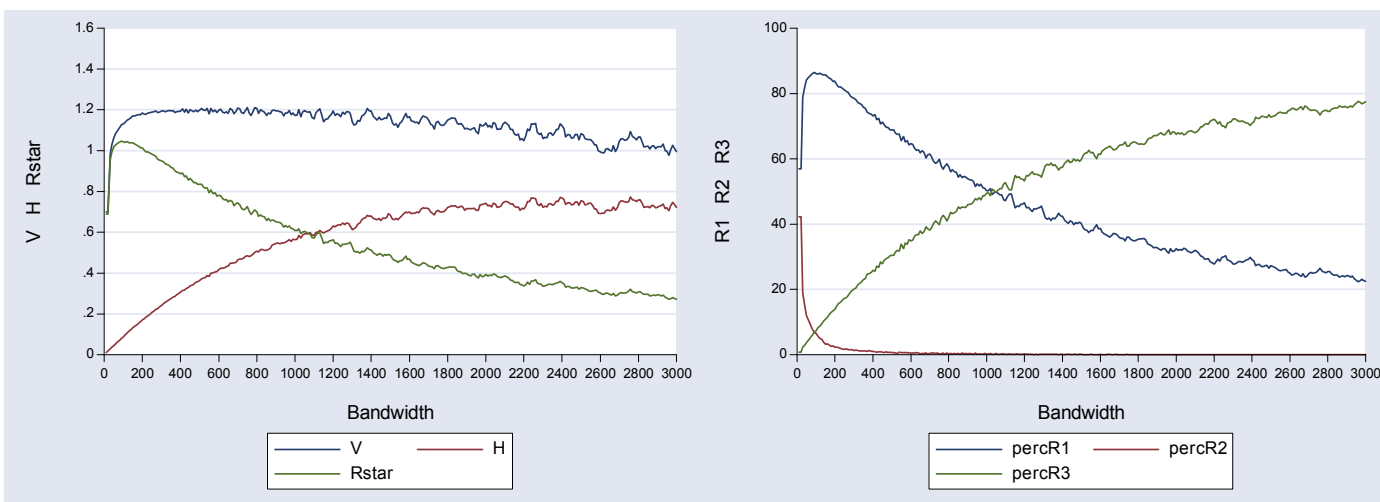

$\S_{m(1)}$ means Cutler scale $\alpha=0.50 \beta=0.65$ and family weight $=1$. 
Figure 7: V/RE, H/RE, $R * / R E$ and $R \%$ decomposition $-m(n)$ Households in $2004^{\$}$

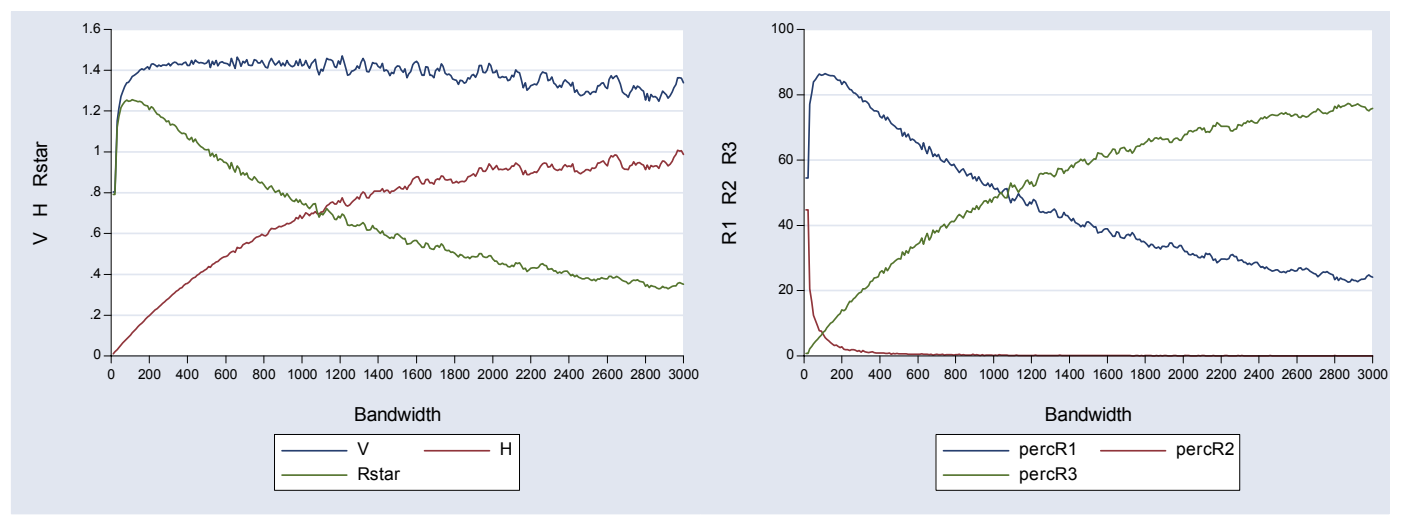

Figure 8: V/RE, H/RE, $R * / R E$ and $R \%$ decomposition - $m(n)$ Households in 2006 \$

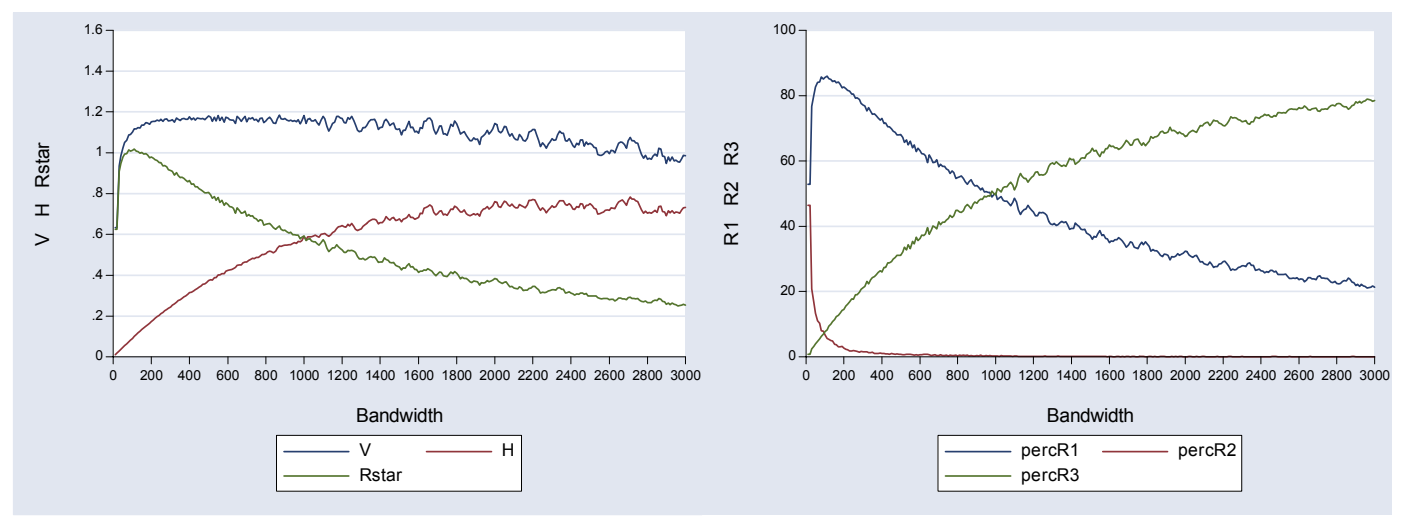

Figure 9: $V / R E, H / R E, R * / R E$ and $R \%$ decomposition - $m(n)$ Households in 2007 \$
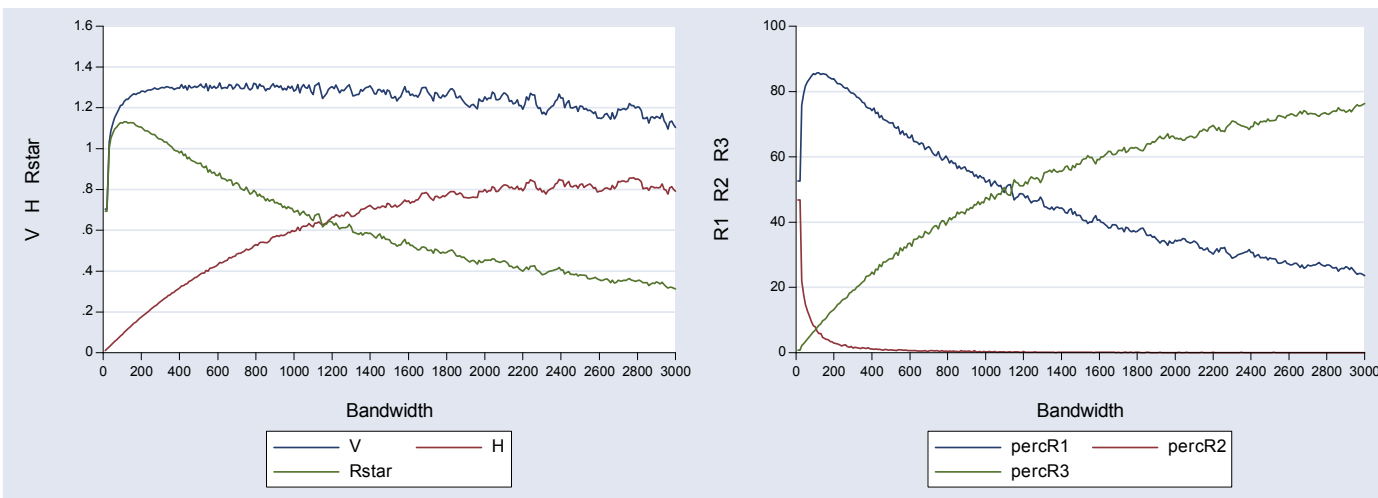

$\$ m(n)$ means Cutler scale $\alpha=0.50 \beta=0.65$ and family weight $=N_{h}$. 
The horizontal effect $\frac{H}{R E}$ is always monotonically increasing (in absolute value): when bigger bandwidths are considered $\frac{G_{y-T}^{W}}{R E}$ rises quicker than $\frac{G_{y}^{W}}{R E}$. If we consider $\frac{R^{*}}{R E}$, it initially increases (in absolute value) up to $w=100$, then monotonically decreases. Analogously to $\frac{V}{R E}, \frac{H}{R E}$ and $\frac{R^{*}}{R E}$ plots becomes irregular as they depart from the axes origin: they take the same irregular wave contour as $\frac{V}{R E}$.

Turning to the decomposition of the Plotnick index, $R_{2}=C_{y-T}^{*}-C_{y-T}^{\circ}$ becomes close to zero for bandwidths bigger than 400: this means that for bandwidths larger than 400 , group average incomes do not present significant rerankings due to taxation.

The transvariation index among groups $R_{1}=G_{y-T}-C_{y-T}^{*}=R^{*}$ decreases and the horizontal inequity due to the reranking within groups $R_{3}=C_{y-T}^{\circ}-C_{y-T}$ increases.

In looking at graphs which represent $R$ decompositions, we observe the same phenomenon we observed for $R E$ decompositions: line becomes more irregular when departing from the axes origin.

Van de Ven, Creedy and Lambert (2001) suggest to choose the optimal bandwidth in correspondence to the maximum value assumed by $\frac{V}{R E}$. Looking at Figures 1-9 we observe that it is not so immediate to individuate an optimal point by the above reported criterion. In correspondence of bandwidths increasing by 10 euro up to $3,000 \frac{V}{R E}$ is not always strictly increasing with respect to the bandwidth, so that the optimal bandwidth should be chosen considering at the same time $\frac{V}{R E}$ trend and its local steadiness.

Moreover, in the spirit of Aronson-Johnson-Lambert's idea, we suggest that the optimal value for $\frac{V}{R E}$ should be chosen when there is no significant reranking for group average incomes, that is when $\frac{R_{2}}{R}$ is almost zero; a considerable value for $\frac{R_{2}}{R}$ may 
make unreliable the value of $V=G_{y}^{B}-G_{y-T}^{B}$ itself if calculated on two average income parades which do not match.

In order to draw $\frac{V}{R E}$ trend and to evaluate its local steadiness, we fitted ${ }^{7} \frac{V}{R E}$ curves represented in Figures 1-9 as a function of the group bandwidths by nonparametric regressions: the kernel function adopted was the uniform with a smoothing bandwidth large about 260 euro (130 at the beginning and at the end). The observed curves, together with the fitted curves, grouped for individual nominal incomes and family equivalent incomes (either with weight 1 and weight equal each family component number), are reported in Figures 10-12.

We can confirm that between bandwidth 250 and 900 euro, $\frac{V}{R E}$ presents a sequence of local maximum points which show a relative stability.

Table 1: The root mean square errors in fitting $(V / R E) \times 100$

\begin{tabular}{lccc}
\hline \hline & $\begin{array}{c}\mathbf{0 - 2 5 0} \\
\text { bandwidths }\end{array}$ & $\begin{array}{c}\mathbf{2 5 1 - 9 0 0} \\
\text { bandwidths }\end{array}$ & $\begin{array}{c}\mathbf{9 0 1 - 3 , 0 0 0} \\
\text { bandwidths }\end{array}$ \\
\hline \hline Individual nominal incomes 2004 & 0.06908 & 0.00954 & 0.04552 \\
\hline Individual nominal incomes 2006 & 0.09256 & 0.01260 & 0.05576 \\
\hline Individual nominal incomes 2007 & 0.08823 & 0.01277 & 0.05310 \\
\hline$m(1)$ Family equivalent incomes 2004 & 0.10936 & 0.01098 & 0.02429 \\
\hline$m(1)$ Family equivalent incomes 2006 & 0.09637 & 0.00881 & 0.02316 \\
\hline$m(1)$ Family equivalent incomes 2007 & 0.10090 & 0.00883 & 0.02062 \\
\hline$m(n)$ Family equivalent incomes 2004 & 0.12891 & 0.01137 & 0.02568 \\
\hline$m(n)$ Family equivalent incomes 2006 & 0.10865 & 0.00912 & 0.02310 \\
\hline$m(n)$ Family equivalent incomes 2007 & 0.11936 & 0.00948 & 0.02145 \\
\hline
\end{tabular}

Source: Own elaborations.

In the 250-900 interval the root mean square errors of the non-parametric fitting are definitely lower both than those calculated for bandwidths less than 250 and than those

\footnotetext{
${ }^{7}$ Non parametric regressions were performed applying the econometric package SHAZAM 10.

${ }^{10}$ Differences between pair of indexes were tested according to the here described procedure: (i) assuming normality by the test function $t=(\operatorname{Index} 1-\operatorname{Index} 2) / \sqrt{\operatorname{se}\{\operatorname{Index} 1\}^{2}+\operatorname{se}\{\operatorname{Index} 2\}^{2}-2 \cdot \operatorname{se}\{\operatorname{Index} 1\} \cdot \operatorname{se}\{\operatorname{Index} 2\}}$ having estimated standard errors $\operatorname{se}\{$ Index 1$\}$ and $\operatorname{se}\{$ Index 2$\}$ by 200 bootstrap replications. Notice that in the above test function correlation 1 is assumed between Index 1 and Index2, which gives the maximum absolute value for $t$; (ii) in all cases $t$ rejects index equality, we estimated $95 \%$ percentile intervals and $s e\{$ Index $1-$ Index 2$\}$ for $($ Index $1-$ Index 2$)$ by 200 bootstrap replications and accepted index equality whenever percentile intervals included 0 and $t=(\operatorname{Index} 1-\operatorname{Index} 2) / \operatorname{se}\{$ Index $1-\operatorname{Index} 2\}$ was not significantly different from zero under normality. Procedure (ii) was always applied for individuals and just in 2006 for $m(1)$ families. Incidentally we observe that bootstrap $95 \%$ percentile intervals are not so different from normal $95 \%$ normal intervals, calculated by bootstrap standard error estimates.
} 
calculated for bandwidths bigger than 900; they are 7-10 times lower than the former ones and 2-4 times lower than the latter ones (Table 1).

Figure 10: $(V / R E) \times 100-$ Actual and fitted values for Individuals

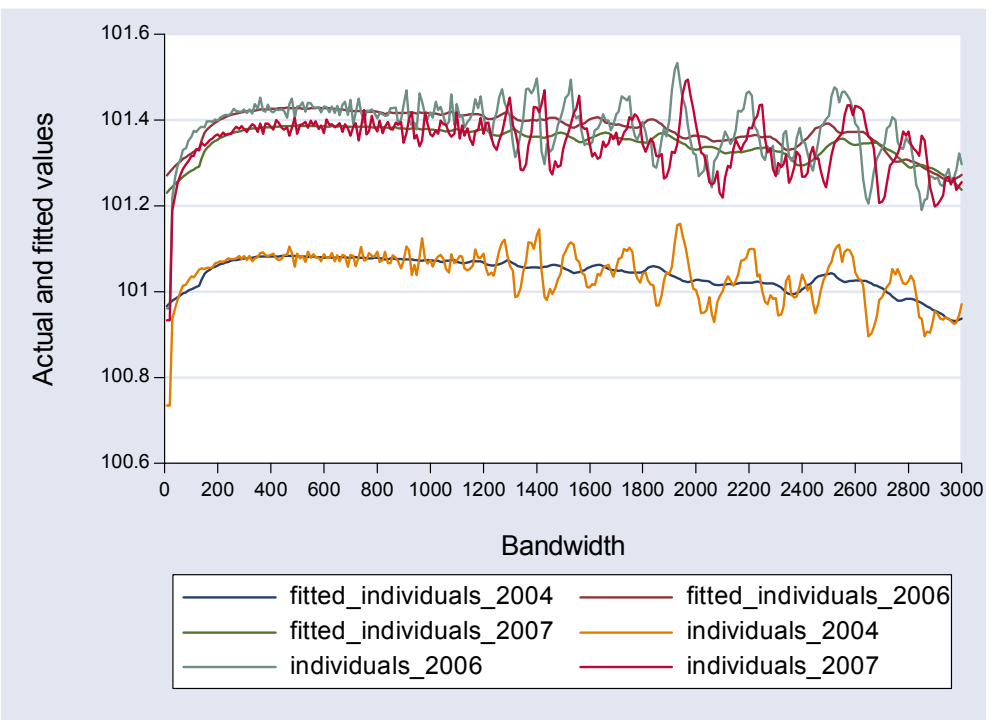

Figure 11: $(V / R E) \times 100$ - Actual and fitted values for $m(1)$ Households

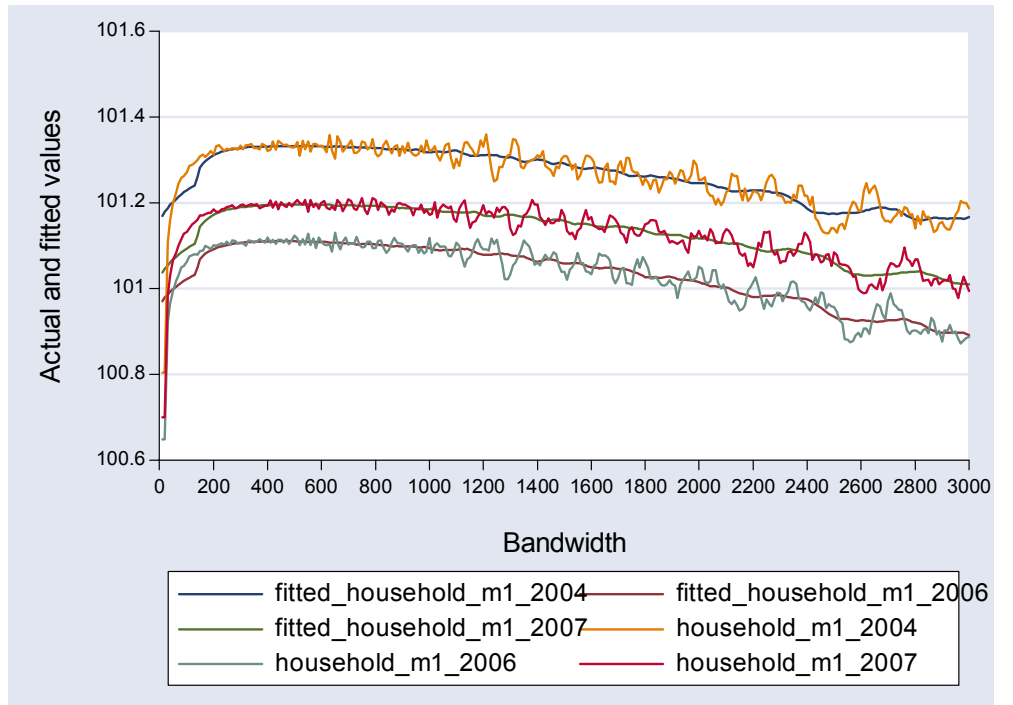

It is worth to stress that the phenomenon that causes an unsatisfactory fitting in the bandwidth set $0-250$, are quite different from those that makes unsatisfactory the fitting in the bandwidth set 901-3,000: in the former set a strongly increasing trend for $\frac{V}{R E}$ makes difficult for a function of the sole bandwidth to follow the actual points. In the 
latter differences between the fitted line and the actual ones are due to the presence of irregular waves which becomes larger in correspondence of larger bandwidths.

Figure 12: $(V / R E) \times 100$ - Actual and fitted values for $m(n)$ Households

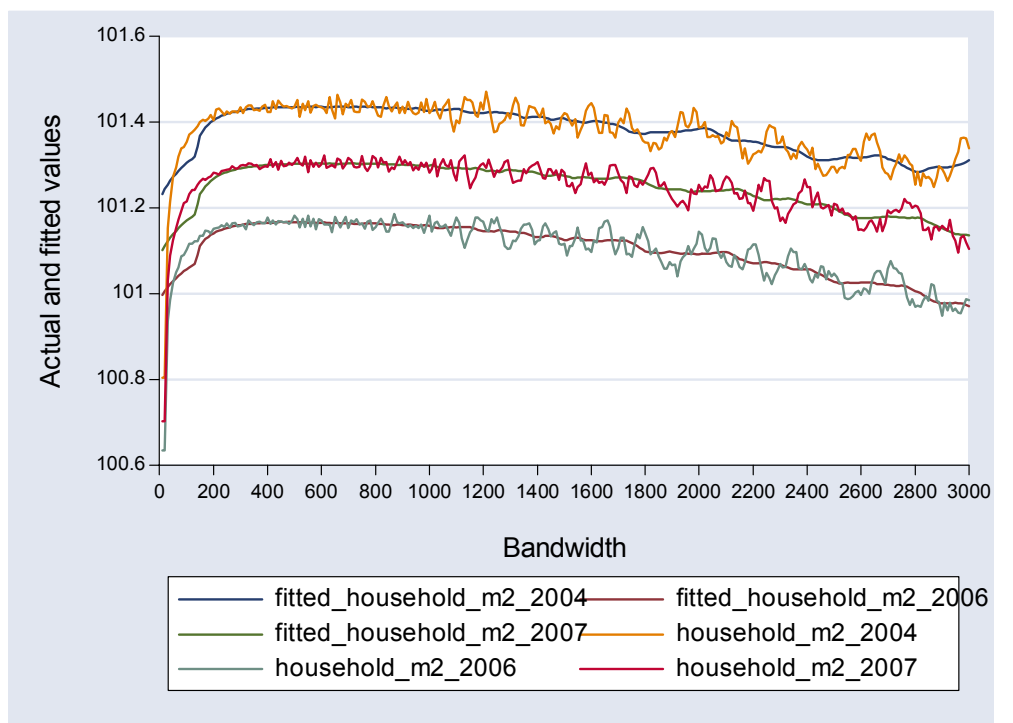

When $w>450$ the percentage of $R_{2}$ on $R$ becomes lower than $1 \%$ : we can then conclude that, for bandwidths larger than 450 euro, post-tax income parades show a reranking for average group incomes quite insignificant.

This is a desirable property to be satisfied. Tables 2-7 report the values for $R E$ and $R$ decompositions, evaluated at bandwidths 100, 700 and 2,000; together with punctual values, the table reports $95 \%$ confidence intervals obtained by 2,000 bootstrap replications percentiles. We can observe that the confidence intervals for $\frac{V}{R E}$ present large overlaps among the three bandwidths; if we perform tests for equality of $\frac{V}{R E}$ at 100-700 and 700-2,000 bandwidth at 5\% significance we always accept equality for all tax systems and all income distributions ${ }^{10}$. The same does not happen for $\frac{R_{2}}{R}$ : here confidence intervals associated to 100 euro bandwidth do not overlap with confidence intervals for 700 and 2,000 euro bandwidth ${ }^{11}$.

\footnotetext{
${ }^{11}$ When $95 \%$ confidence intervals do not overlap, index inequality is rejected at a significance level even lower than $5 \%$.
} 
Table 2: $R E$ decomposition - Individuals

(bootstrap estimated $95 \%$ confidence intervals in parentheses-2,000 replications)

\begin{tabular}{|c|c|c|c|c|}
\hline & & 2004 & 2006 & 2007 \\
\hline \multirow[t]{2}{*}{$\% R E / G_{y}$} & & $\begin{array}{c}14.370 \\
(14.109-14.620) \\
\end{array}$ & $\begin{array}{c}13.453 \\
(13.211-13.684) \\
\end{array}$ & $\begin{array}{c}14.003 \\
(13.747-14.243) \\
\end{array}$ \\
\hline & bandwidth & & $\% R E$ & \\
\hline \multirow{3}{*}{$V$} & 100 & $\begin{array}{c}101.036 \\
(100.649-101.424)\end{array}$ & $\begin{array}{c}101.361 \\
(100.932-101.835)\end{array}$ & $\begin{array}{c}101.317 \\
(100.894-101.774)\end{array}$ \\
\hline & 700 & $\begin{array}{c}101.086 \\
(100.632-101.516) \\
\end{array}$ & $\begin{array}{c}101.448 \\
(100.949-101.953) \\
\end{array}$ & $\begin{array}{c}101.364 \\
(100.902-101.866) \\
\end{array}$ \\
\hline & 2000 & $\begin{array}{c}101.004 \\
(100.538-101.492) \\
\end{array}$ & $\begin{array}{c}101.320 \\
(100.818-101.892) \\
\end{array}$ & $\begin{array}{c}101.390 \\
(100.871-101.950) \\
\end{array}$ \\
\hline \multirow{3}{*}{$H$} & 100 & $\begin{array}{c}0.076 \\
(-0.317-0.461)\end{array}$ & $\begin{array}{c}0.091 \\
(-0.379-0.562)\end{array}$ & $\begin{array}{c}0.087 \\
(-0.347-0.510)\end{array}$ \\
\hline & 700 & $\begin{array}{c}0.407 \\
(-0.041-0.854) \\
\end{array}$ & $\begin{array}{c}0.502 \\
(0.027-1.002)\end{array}$ & $\begin{array}{c}0.471 \\
(0.002-0.939)\end{array}$ \\
\hline & 2000 & $\begin{array}{c}0.671 \\
(0.208-1.154)\end{array}$ & $\begin{array}{c}0.855 \\
(0.291-1.388)\end{array}$ & $\begin{array}{c}0.898 \\
(0.398-1.421)\end{array}$ \\
\hline \multirow{3}{*}{$R^{*}$} & 100 & $\begin{array}{c}0.960 \\
(0.894-1.032) \\
\end{array}$ & $\begin{array}{c}1.270 \\
(1.182-1.370) \\
\end{array}$ & $\begin{array}{c}1.230 \\
(1.139-1.326) \\
\end{array}$ \\
\hline & 700 & $\begin{array}{c}0.679 \\
(0.629-0.734)\end{array}$ & $\begin{array}{c}0.945 \\
(0.875-1.020)\end{array}$ & $\begin{array}{c}0.893 \\
(0.823-0.967)\end{array}$ \\
\hline & 2000 & $\begin{array}{c}0.333 \\
(0.300-0.367) \\
\end{array}$ & $\begin{array}{c}0.465 \\
(0.418-0.513) \\
\end{array}$ & $\begin{array}{c}0.492 \\
(0.445-0.543) \\
\end{array}$ \\
\hline
\end{tabular}

Source: Own elaborations.

Table 3: $\boldsymbol{R}$ decomposition - Individuals

(bootstrap estimated $95 \%$ confidence intervals in parentheses-2,000 replications)

\begin{tabular}{|c|c|c|c|c|}
\hline & & 2004 & 2006 & 2007 \\
\hline \multirow[t]{2}{*}{$\overline{\%} \% \mathrm{R} / \mathrm{RE}$} & & 1.087 & 1.432 & 1.389 \\
\hline & bandwidth & & $\% R$ & \\
\hline \multirow{3}{*}{$R_{1}$} & 100 & $\begin{array}{c}88.286 \\
(87.185-89.345)\end{array}$ & $\begin{array}{c}88.700 \\
(87.547-89.834)\end{array}$ & $\begin{array}{c}88.556 \\
(87.388-89.720)\end{array}$ \\
\hline & 700 & $\begin{array}{c}62.450 \\
(61.300-63.610)\end{array}$ & $\begin{array}{c}66.029 \\
(64.977-67.140)\end{array}$ & $\begin{array}{c}64.250 \\
(63.112-65.369)\end{array}$ \\
\hline & 2000 & $\begin{array}{c}30.589 \\
(28.932-32.304)\end{array}$ & $\begin{array}{c}32.458 \\
(30.757-34.205)\end{array}$ & $\begin{array}{c}35.412 \\
(33.666-37.115)\end{array}$ \\
\hline \multirow{3}{*}{$R_{2}$} & 100 & $\begin{array}{c}4.596 \\
(3.482-5.620)\end{array}$ & $\begin{array}{c}4.816 \\
(3.694-6.052)\end{array}$ & $\begin{array}{c}5.308 \\
(4.089-6.514)\end{array}$ \\
\hline & 700 & $\begin{array}{c}0.067 \\
(0.022-0.135)\end{array}$ & $\begin{array}{c}0.035 \\
(0.000-0.085)\end{array}$ & $\begin{array}{c}0.034 \\
(0.000-0.089)\end{array}$ \\
\hline & 2000 & $\begin{array}{c}0.000 \\
(0.000-0.046)\end{array}$ & $\begin{array}{c}0.000 \\
(0.000-0.018)\end{array}$ & $\begin{array}{c}0.017 \\
(0.000-0.018)\end{array}$ \\
\hline \multirow{3}{*}{$R_{3}$} & 100 & $\begin{array}{c}7.112 \\
(6.839-7.403)\end{array}$ & $\begin{array}{c}6.485 \\
(6.245-6.759)\end{array}$ & $\begin{array}{c}6.136 \\
(5.887-6.375)\end{array}$ \\
\hline & 700 & $\begin{array}{c}37.483 \\
(36.219-38.719)\end{array}$ & $\begin{array}{c}33.936 \\
(32.851-35.032)\end{array}$ & $\begin{array}{c}35.717 \\
(34.569-36.874)\end{array}$ \\
\hline & 2000 & $\begin{array}{c}69.411 \\
(67.600-71.197)\end{array}$ & $\begin{array}{c}67.542 \\
(65.799-69.292)\end{array}$ & $\begin{array}{c}64.571 \\
(62.909-66.333)\end{array}$ \\
\hline
\end{tabular}


Table 4: $R E$ decomposition - $m(1)$ Households

(bootstrap estimated 95\% confidence intervals in parentheses-2,000 replications)

\begin{tabular}{|c|c|c|c|c|}
\hline & & 2004 & 2006 & 2007 \\
\hline \multirow[t]{2}{*}{$\% R E / G_{y}$} & & $\begin{array}{c}13.927 \\
(13.545-14.287) \\
\end{array}$ & $\begin{array}{c}13.328 \\
(13.002-13.634) \\
\end{array}$ & $\begin{array}{c}13.852 \\
(13.510-14.187) \\
\end{array}$ \\
\hline & bandwidth & & $\% R E$ & \\
\hline \multirow{3}{*}{$V$} & 100 & $\begin{array}{c}101.270 \\
(100.625-101.975)\end{array}$ & $\begin{array}{c}101.062 \\
(100.357-101.740)\end{array}$ & $\begin{array}{c}101.132 \\
(100.497-101.777)\end{array}$ \\
\hline & 700 & $\begin{array}{c}101.335 \\
(100.528-102.197) \\
\end{array}$ & $\begin{array}{c}101.102 \\
(100.225-101.900) \\
\end{array}$ & $\begin{array}{c}101.209 \\
(100.393-102.082) \\
\end{array}$ \\
\hline & 2000 & $\begin{array}{c}101.253 \\
(100.364-102.187) \\
\end{array}$ & $\begin{array}{c}101.052 \\
(100.158-101.925) \\
\end{array}$ & $\begin{array}{c}101.135 \\
(100.247-102.117) \\
\end{array}$ \\
\hline \multirow{3}{*}{$H$} & 100 & $\begin{array}{c}0.100 \\
(-0.544-0.756)\end{array}$ & $\begin{array}{c}0.090 \\
(-0.553-0.771)\end{array}$ & $\begin{array}{c}0.089 \\
(-0.588-0.776)\end{array}$ \\
\hline & 700 & $\begin{array}{c}0.525 \\
(-0.284-1.362)\end{array}$ & $\begin{array}{c}0.456 \\
(-0.379-1.251)\end{array}$ & $\begin{array}{c}0.465 \\
(-0.376-1.330)\end{array}$ \\
\hline & 2000 & $\begin{array}{c}0.832 \\
(-0.108-1.778)\end{array}$ & $\begin{array}{c}0.706 \\
(-0.213-1.616)\end{array}$ & $\begin{array}{c}0.742 \\
(-0.115-1.647)\end{array}$ \\
\hline \multirow{3}{*}{$R^{*}$} & 100 & $\begin{array}{c}1.170 \\
(1.078-1.276) \\
\end{array}$ & $\begin{array}{c}0.972 \\
(0.897-1.056) \\
\end{array}$ & $\begin{array}{c}1.043 \\
(0.963-1.138) \\
\end{array}$ \\
\hline & 700 & $\begin{array}{c}0.811 \\
(0.736-0.888)\end{array}$ & $\begin{array}{c}0.646 \\
(0.591-0.713)\end{array}$ & $\begin{array}{c}0.744 \\
(0.677-0.817)\end{array}$ \\
\hline & 2000 & $\begin{array}{c}0.421 \\
(0.375-0.472) \\
\end{array}$ & $\begin{array}{c}0.346 \\
(0.308-0.389) \\
\end{array}$ & $\begin{array}{c}0.394 \\
(0.350-0.440) \\
\end{array}$ \\
\hline
\end{tabular}

Source: Own elaborations.

Table 5: $R$ decomposition - $m(1)$ Households

(bootstrap estimated $95 \%$ confidence intervals in parentheses-2,000 replications)

\begin{tabular}{|c|c|c|c|c|}
\hline & & 2004 & 2006 & 2007 \\
\hline \multirow[t]{2}{*}{$\% R / R E$} & & $\begin{array}{c}1.349 \\
(1.240-1.473) \\
\end{array}$ & $\begin{array}{c}1.126 \\
(1.040-1.222) \\
\end{array}$ & $\begin{array}{c}1.211 \\
(1.118-1.322) \\
\end{array}$ \\
\hline & bandwidth & & $\% R$ & \\
\hline \multirow{3}{*}{$R_{1}$} & 100 & $\begin{array}{c}86.762 \\
(85.374-88.090)\end{array}$ & $\begin{array}{c}86.323 \\
(84.894-87.640)\end{array}$ & $\begin{array}{c}86.068 \\
(84.557-87.439)\end{array}$ \\
\hline & 700 & $\begin{array}{c}60.107 \\
(58.683-61.488)\end{array}$ & $\begin{array}{c}57.380 \\
(55.815-58.872)\end{array}$ & $\begin{array}{c}61.408 \\
(59.901-62.866)\end{array}$ \\
\hline & 2000 & $\begin{array}{c}31.219 \\
(29.170-33.110)\end{array}$ & $\begin{array}{c}30.701 \\
(28.604-32.850)\end{array}$ & $\begin{array}{c}32.502 \\
(30.458-34.562) \\
\end{array}$ \\
\hline \multirow{3}{*}{$R_{2}$} & 100 & $\begin{array}{c}5.710 \\
(4.335-7.136)\end{array}$ & $\begin{array}{c}5.562 \\
(4.178-7.010)\end{array}$ & $\begin{array}{c}6.596 \\
(5.109-8.130)\end{array}$ \\
\hline & 700 & $\begin{array}{c}0.474 \\
(0.154-0.906) \\
\end{array}$ & $\begin{array}{c}0.361 \\
(0.025-0.721)\end{array}$ & $\begin{array}{c}0.359 \\
(0.042-0.744) \\
\end{array}$ \\
\hline & 2000 & $\begin{array}{c}0.040 \\
(0.000-0.099) \\
\end{array}$ & $\begin{array}{c}0.048 \\
(0.000-0.124) \\
\end{array}$ & $\begin{array}{c}0.042 \\
(0.000-0.084) \\
\end{array}$ \\
\hline \multirow{3}{*}{$R_{3}$} & 100 & $\begin{array}{c}7.528 \\
(7.181-7.859) \\
\end{array}$ & $\begin{array}{c}8.115 \\
(7.733-8.466) \\
\end{array}$ & $\begin{array}{c}7.336 \\
(6.997-7.676) \\
\end{array}$ \\
\hline & 700 & $\begin{array}{c}39.419 \\
(38.018-40.862)\end{array}$ & $\begin{array}{c}42.259 \\
(40.771-43.895)\end{array}$ & $\begin{array}{c}38.232 \\
(36.846-39.750)\end{array}$ \\
\hline & 2000 & $\begin{array}{c}688.741 \\
(66.896-70.575)\end{array}$ & $\begin{array}{c}69.251 \\
(67.274-71.328)\end{array}$ & $\begin{array}{c}67.463 \\
(65.447-69.529)\end{array}$ \\
\hline
\end{tabular}


Table 6: $R E$ decomposition - $m(n)$ Households

(bootstrap estimated $95 \%$ confidence intervals in parentheses-2,000 replications)

\begin{tabular}{|c|c|c|c|c|}
\hline & & 2004 & 2006 & 2007 \\
\hline \multirow[t]{2}{*}{$\% R E / G_{y}$} & & $\begin{array}{c}13.451 \\
(13.045-13.824) \\
\end{array}$ & $\begin{array}{c}13.035 \\
(12.674-13.373) \\
\end{array}$ & $\begin{array}{c}13.559 \\
(13.197-13.918) \\
\end{array}$ \\
\hline & bandwidth & & $\% R E$ & \\
\hline \multirow{3}{*}{$V$} & 100 & $\begin{array}{c}101.354 \\
(100.654-102.125) \\
\end{array}$ & $\begin{array}{c}101.104 \\
(100.366-101.850) \\
\end{array}$ & $\begin{array}{c}101.215 \\
(100.493-102.012)\end{array}$ \\
\hline & 700 & $\begin{array}{c}101.435 \\
(100.478-102.427)\end{array}$ & $\begin{array}{c}101.153 \\
(100.197-102.141)\end{array}$ & $\begin{array}{c}101.316 \\
(100.405-102.287)\end{array}$ \\
\hline & 2000 & $\begin{array}{c}101.387 \\
(100.279-102.506)\end{array}$ & $\begin{array}{c}101.145 \\
(100.107-102.176)\end{array}$ & $\begin{array}{c}101.254 \\
(100.183-102.298)\end{array}$ \\
\hline \multirow{3}{*}{$H$} & 100 & $\begin{array}{c}0.103 \\
(-0.632-0.829) \\
\end{array}$ & $\begin{array}{c}0.092 \\
(-0.641-0.868) \\
\end{array}$ & $\begin{array}{c}0.091 \\
(-0.682-0.849) \\
\end{array}$ \\
\hline & 700 & $\begin{array}{c}0.548 \\
(-0.392-1.560) \\
\end{array}$ & $\begin{array}{c}0.464 \\
(-0.494-1.390) \\
\end{array}$ & $\begin{array}{c}0.483 \\
(-0.457-1.472) \\
\end{array}$ \\
\hline & 2000 & $\begin{array}{c}0.913 \\
(-0.127-1.984) \\
\end{array}$ & $\begin{array}{c}0.761 \\
(-0.228-1.807) \\
\end{array}$ & $\begin{array}{c}0.799 \\
(-0.306-1.827) \\
\end{array}$ \\
\hline \multirow{3}{*}{$R^{*}$} & 100 & $\begin{array}{c}1.251 \\
(1.141-1.372)\end{array}$ & $\begin{array}{c}1.012 \\
(0.927-1.107)\end{array}$ & $\begin{array}{c}1.124 \\
(1.028-1.231)\end{array}$ \\
\hline & 700 & $\begin{array}{c}0.887 \\
(0.802-0.985) \\
\end{array}$ & $\begin{array}{c}0.688 \\
(0.623-0.757) \\
\end{array}$ & $\begin{array}{c}0.833 \\
(0.756-0.924)\end{array}$ \\
\hline & 2000 & $\begin{array}{c}0.474 \\
(0.417-0.538) \\
\end{array}$ & $\begin{array}{c}0.384 \\
(0.338-0.436) \\
\end{array}$ & $\begin{array}{c}0.454 \\
(0.397-0.517) \\
\end{array}$ \\
\hline
\end{tabular}

Source: Own elaborations.

Table 7: $\boldsymbol{R}$ decomposition $-\boldsymbol{m}(\boldsymbol{n})$ Households

(bootstrap estimated $95 \%$ confidence intervals in parentheses-2,000 replications)

\begin{tabular}{|c|c|c|c|c|}
\hline & & 2004 & 2006 & 2007 \\
\hline \multirow[t]{2}{*}{$\% R / R E$} & & & & 1.320 \\
\hline & Bandwidth & & $\% R$ & \\
\hline \multirow{3}{*}{$R_{1}$} & 100 & $\begin{array}{c}86.104 \\
(84.458-87.601)\end{array}$ & $\begin{array}{c}85.539 \\
(84.004-87.080)\end{array}$ & $\begin{array}{c}85.167 \\
(83.444-86.850\end{array}$ \\
\hline & 700 & $\begin{array}{c}61.061 \\
(59.402-62.665)\end{array}$ & $\begin{array}{c}58.196 \\
(56.371-59.916)\end{array}$ & $\begin{array}{c}63.098 \\
(61.398-64.784)\end{array}$ \\
\hline & 2000 & $\begin{array}{c}32.654 \\
(30.413-35.078)\end{array}$ & $\begin{array}{c}32.431 \\
(30.020-34.974)\end{array}$ & $\begin{array}{c}34.430 \\
(32.093-36.944)\end{array}$ \\
\hline \multirow{3}{*}{$R_{2}$} & 100 & $\begin{array}{c}6.688 \\
(5.117-8.329)\end{array}$ & $\begin{array}{c}6.689 \\
(5.044-8.333)\end{array}$ & $\begin{array}{c}7.974 \\
(6.130-9.783)\end{array}$ \\
\hline & 700 & $\begin{array}{c}0.538 \\
(0.138-0.974) \\
\end{array}$ & $\begin{array}{c}0.447 \\
(0.069-0.864) \\
\end{array}$ & $\begin{array}{c}0.518 \\
(0.119-0.947) \\
\end{array}$ \\
\hline & 2000 & $\begin{array}{c}0.058 \\
(0.000-0.106)\end{array}$ & $\begin{array}{c}0.071 \\
(0.000-0.173)\end{array}$ & $\begin{array}{c}0.040 \\
(0.000-0.105)\end{array}$ \\
\hline \multirow{3}{*}{$R_{3}$} & 100 & $\begin{array}{c}7.207 \\
(6.824-7.600)\end{array}$ & $\begin{array}{c}7.772 \\
(7.374-8.198)\end{array}$ & $\begin{array}{c}6.858 \\
(6.464-7.286)\end{array}$ \\
\hline & 700 & $\begin{array}{c}38.401 \\
(36.834-40.067) \\
\end{array}$ & $\begin{array}{c}41.357 \\
(39.537-43.154) \\
\end{array}$ & $\begin{array}{c}36.384 \\
(34.703-38.099) \\
\end{array}$ \\
\hline & 2000 & $\begin{array}{c}67.288 \\
(65.088-69.541)\end{array}$ & $\begin{array}{c}67.499 \\
(65.021-69.892)\end{array}$ & $\begin{array}{c}65.530 \\
(63.119-68.009)\end{array}$ \\
\hline
\end{tabular}


Even if we cannot always accept equality between $\frac{R_{2}}{R}$ associated to 700 and 2,000 bandwidths, observing that when fixing bandwidth at 700 euro, $R_{2}$ confidence intervals never exceed $1 \%$ of $R$, we conclude that such a bandwidth is large enough to perform tax law evaluations by the Bank of Italy data base. The tables show that the point estimates are quite reliable but for $\frac{H}{R E}$ : this index presents quite relative confidence intervals, which include negative values. This implies that somewhere Gini index within groups may be lower after taxation then before.

\section{Evaluating personal income tax reforms}

In this section we consider the redistributive effect $R E$ and the reranking effect $R$ of the Italian PIT, with respect to both individual incomes and equivalent incomes.

Equivalent incomes are evaluated by nine different Cutler scale levels: the scales are obtained by combining three different values for $\beta(0.50,0.65,0.80)$ and three different values for $\alpha(0.30,0.50,0.70)$. The resulting incomes are then weighed by the corresponding values of the scale itself. We use this special weighting version for two reasons: the total amount of both the gross and the net incomes are not affected with respect to the original nominal values; the way of determining the weights lies between the ones used for the $m(1)$ and the $m(n)$ methods.

Together with $R E$ and $R$, we present their respective components $V, H, R^{*}$ and $R_{1}=R^{*}, R_{2}, R_{3}$; these components are evaluated at the 700 euro bandwidth, which we concluded to be optimal in the previous section. Due to the fact that inequality measures depend on scales, all indexes are reported as percentage of the Gini index associated to the corresponding pre-tax equivalent income parade, in order to get comparable measures. All results are summarized in Table 8.

In what it concerns family equivalent incomes, we observe that the actual redistributive effect $R E$ ranges from 12.83 to 13.76 percent point of the pre-tax Gini index; the potential $V$ ranges from 13.00 to 13.93 ; both $R E$ and $V$ presents maximum values in 2007 and minimum in 2006. 
Table 8: $R E$ and $R$ decomposition - households (weight=scale) and individuals

(bootstrap estimated standard errors in parentheses: 200 replications)

\begin{tabular}{|c|c|c|c|c|c|c|c|c|c|}
\hline \multicolumn{2}{|c|}{$\begin{array}{l}\text { Cutler scale } \\
\text { parameters }\end{array}$} & \multirow[t]{2}{*}{ Year } & \multirow[t]{2}{*}{$\left(R E / G_{y}\right) \%$} & \multirow[t]{2}{*}{$\left(V / G_{y}\right) \%$} & \multirow[t]{2}{*}{$\left(H / G_{y}\right) \%$} & \multirow{2}{*}{$\begin{array}{l}\left(R^{*} / G_{y}\right) \% \\
\left(R_{1} / G_{y}\right) \%\end{array}$} & \multirow[t]{2}{*}{$\left(R / G_{y}\right) \%$} & \multirow[t]{2}{*}{$\left(R_{2} / G_{y}\right) \%$} & \multirow[t]{2}{*}{$\left(R_{3} / G_{y}\right) \%$} \\
\hline$\alpha$ & $\beta$ & & & & & & & & \\
\hline \multirow{3}{*}{0.30} & \multirow{3}{*}{0.50} & 2004 & $\begin{array}{c}13.4886 \\
(.1751) \\
\end{array}$ & $\begin{array}{l}13.6957 \\
(.1756) \\
\end{array}$ & $\begin{array}{c}.0707 \\
(.0589) \\
\end{array}$ & $\begin{array}{c}.1364 \\
(.0051) \\
\end{array}$ & $\begin{array}{c}.2075 \\
(.0065) \\
\end{array}$ & $\begin{array}{c}.0006 \\
(.0002) \\
\end{array}$ & $\begin{array}{c}.0705 \\
(.0023) \\
\end{array}$ \\
\hline & & 2006 & $\begin{array}{l}12.9990 \\
(.1725)\end{array}$ & $\begin{array}{l}13.1710 \\
(.1594)\end{array}$ & $\begin{array}{c}.0613 \\
(.0618)\end{array}$ & $\begin{array}{l}.1106 \\
(.0042)\end{array}$ & $\begin{array}{c}.1733 \\
(.0062)\end{array}$ & $\begin{array}{c}.0008 \\
(.0004)\end{array}$ & $\begin{array}{c}.0619 \\
(.0020)\end{array}$ \\
\hline & & 2007 & $\begin{array}{l}13.5126 \\
(.1474) \\
\end{array}$ & $\begin{array}{l}13.7031 \\
(.1460) \\
\end{array}$ & $\begin{array}{c}.0636 \\
(.0601) \\
\end{array}$ & $\begin{array}{c}.1269 \\
(.0049) \\
\end{array}$ & $\begin{array}{c}.1927 \\
(.0071) \\
\end{array}$ & $\begin{array}{c}.0009 \\
(.0004) \\
\end{array}$ & $\begin{array}{c}.0649 \\
(.0022) \\
\end{array}$ \\
\hline \multirow{3}{*}{0.30} & \multirow{3}{*}{0.65} & 2004 & $\begin{array}{l}13.7295 \\
(.1826) \\
\end{array}$ & $\begin{array}{l}13.9255 \\
(.1874) \\
\end{array}$ & $\begin{array}{c}.0748 \\
(.0633) \\
\end{array}$ & $\begin{array}{c}.1212 \\
(.0054) \\
\end{array}$ & $\begin{array}{c}.1985 \\
(.0076) \\
\end{array}$ & $\begin{array}{c}.0010 \\
(.0003) \\
\end{array}$ & $\begin{array}{c}.0763 \\
(.0026) \\
\end{array}$ \\
\hline & & 2006 & $\begin{array}{l}13.2132 \\
(.1637) \\
\end{array}$ & $\begin{array}{l}13.3717 \\
(.1411) \\
\end{array}$ & $\begin{array}{c}.0638 \\
(.0548) \\
\end{array}$ & $\begin{array}{c}.0947 \\
(.0043) \\
\end{array}$ & $\begin{array}{c}.1604 \\
(.0054) \\
\end{array}$ & $\begin{array}{c}.0006 \\
(.0002) \\
\end{array}$ & $\begin{array}{c}.0651 \\
(.0021) \\
\end{array}$ \\
\hline & & 2007 & $\begin{array}{l}13.7587 \\
(.1667) \\
\end{array}$ & $\begin{array}{l}13.9410 \\
(.1533) \\
\end{array}$ & $\begin{array}{c}.0672 \\
(.0657) \\
\end{array}$ & $\begin{array}{c}.1151 \\
(.0055) \\
\end{array}$ & $\begin{array}{c}.1806 \\
(.0061) \\
\end{array}$ & $\begin{array}{c}.0009 \\
(.0004) \\
\end{array}$ & $\begin{array}{c}.0646 \\
(.0022) \\
\end{array}$ \\
\hline \multirow{3}{*}{0.30} & \multirow{3}{*}{0.80} & 2004 & $\begin{array}{c}13.5685 \\
(.1884) \\
\end{array}$ & $\begin{array}{l}13.7748 \\
(.1825) \\
\end{array}$ & $\begin{array}{c}.0843 \\
(.0623) \\
\end{array}$ & $\begin{array}{c}.1220 \\
(.0056) \\
\end{array}$ & $\begin{array}{c}.2076 \\
(.0083) \\
\end{array}$ & $\begin{array}{c}.0005 \\
(.0002) \\
\end{array}$ & $\begin{array}{c}.0852 \\
(.0031) \\
\end{array}$ \\
\hline & & 2006 & $\begin{array}{l}13.1428 \\
(.1753) \\
\end{array}$ & $\begin{array}{l}13.3035 \\
(.1551) \\
\end{array}$ & $\begin{array}{c}.0697 \\
(.0683) \\
\end{array}$ & $\begin{array}{c}.0910 \\
(.0043) \\
\end{array}$ & $\begin{array}{c}.1637 \\
(.0064) \\
\end{array}$ & $\begin{array}{c}.0004 \\
(.0002) \\
\end{array}$ & $\begin{array}{c}.0723 \\
(.0025) \\
\end{array}$ \\
\hline & & 2007 & $\begin{array}{l}13.6634 \\
(.1803) \\
\end{array}$ & $\begin{array}{l}13.8494 \\
(.1516) \\
\end{array}$ & $\begin{array}{c}.0739 \\
(.0617) \\
\end{array}$ & $\begin{array}{c}.1122 \\
(.0057) \\
\end{array}$ & $\begin{array}{c}.1870 \\
(.0068) \\
\end{array}$ & $\begin{array}{c}.0005 \\
(.0003) \\
\end{array}$ & $\begin{array}{c}.0744 \\
(.0026) \\
\end{array}$ \\
\hline \multirow{3}{*}{0.50} & \multirow{3}{*}{0.50} & 2004 & $\begin{array}{c}13.4507 \\
(.1809) \\
\end{array}$ & $\begin{array}{c}13.6556 \\
(.1746) \\
\end{array}$ & $\begin{array}{c}.0704 \\
(.0627) \\
\end{array}$ & $\begin{array}{c}.1345 \\
(.0054) \\
\end{array}$ & $\begin{array}{c}.2047 \\
(.0073) \\
\end{array}$ & $\begin{array}{c}.0006 \\
(.0002) \\
\end{array}$ & $\begin{array}{c}.0696 \\
(.0025) \\
\end{array}$ \\
\hline & & 2006 & $\begin{array}{l}12.9787 \\
(.1583) \\
\end{array}$ & $\begin{array}{c}13.1466 \\
(.1359) \\
\end{array}$ & $\begin{array}{c}.0603 \\
(.0610) \\
\end{array}$ & $\begin{array}{c}.1076 \\
(.0043) \\
\end{array}$ & $\begin{array}{c}.1686 \\
(.0056) \\
\end{array}$ & $\begin{array}{c}.0010 \\
(.0004) \\
\end{array}$ & $\begin{array}{c}.0600 \\
(.0020) \\
\end{array}$ \\
\hline & & 2007 & $\begin{array}{c}13.4800 \\
(.1606) \\
\end{array}$ & $\begin{array}{c}13.6667 \\
(.1464) \\
\end{array}$ & $\begin{array}{c}.0626 \\
(.0610) \\
\end{array}$ & $\begin{array}{c}.1241 \\
(.0049) \\
\end{array}$ & $\begin{array}{c}.1893 \\
(.0066) \\
\end{array}$ & $\begin{array}{c}.0013 \\
(.0005) \\
\end{array}$ & $\begin{array}{c}.0639 \\
(.0023) \\
\end{array}$ \\
\hline \multirow{3}{*}{0.50} & \multirow{3}{*}{0.65} & 2004 & $\begin{array}{l}13.6413 \\
(.1899) \\
\end{array}$ & $\begin{array}{l}13.8365 \\
(.1707) \\
\end{array}$ & $\begin{array}{c}.0752 \\
(.0648) \\
\end{array}$ & $\begin{array}{c}.1200 \\
(.0052) \\
\end{array}$ & $\begin{array}{c}.1974 \\
(.0069) \\
\end{array}$ & $\begin{array}{c}.0010 \\
(.0004) \\
\end{array}$ & $\begin{array}{c}.0764 \\
(.0026) \\
\end{array}$ \\
\hline & & 2006 & $\begin{array}{c}13.1491 \\
(1695) \\
\end{array}$ & $\begin{array}{c}13.3019 \\
(.1486) \\
\end{array}$ & $\begin{array}{c}.0620 \\
(.0625) \\
\end{array}$ & $\begin{array}{c}.0908 \\
(.0037) \\
\end{array}$ & $\begin{array}{c}.1565 \\
(.0052) \\
\end{array}$ & $\begin{array}{c}.0007 \\
(.0003) \\
\end{array}$ & $\begin{array}{c}.0651 \\
(.0023) \\
\end{array}$ \\
\hline & & 2007 & $\begin{array}{c}13.6764 \\
(.1867) \\
\end{array}$ & $\begin{array}{c}13.8550 \\
(.1606) \\
\end{array}$ & $\begin{array}{c}.0665 \\
(.0693) \\
\end{array}$ & $\begin{array}{c}.1121 \\
(.0052) \\
\end{array}$ & $\begin{array}{c}.1788 \\
(.0066) \\
\end{array}$ & $\begin{array}{c}.0008 \\
(.0003) \\
\end{array}$ & $\begin{array}{c}.0658 \\
(.0023) \\
\end{array}$ \\
\hline \multirow{3}{*}{0.50} & \multirow{3}{*}{0.80} & 2004 & $\begin{array}{c}13.4229 \\
(.1852) \\
\end{array}$ & $\begin{array}{l}13.6305 \\
(.1772) \\
\end{array}$ & $\begin{array}{c}.0852 \\
(.0718) \\
\end{array}$ & $\begin{array}{c}.1224 \\
(.0057) \\
\end{array}$ & $\begin{array}{c}.2086 \\
(.0074) \\
\end{array}$ & $\begin{array}{c}.0004 \\
(.0003) \\
\end{array}$ & $\begin{array}{c}.0858 \\
(.0028) \\
\end{array}$ \\
\hline & & 2006 & $\begin{array}{l}13.0269 \\
(.1577)\end{array}$ & $\begin{array}{l}13.1855 \\
(.1509)\end{array}$ & $\begin{array}{c}.0691 \\
(.0658) \\
\end{array}$ & $\begin{array}{c}.0895 \\
(.0044) \\
\end{array}$ & $\begin{array}{c}.1614 \\
(.0057) \\
\end{array}$ & $\begin{array}{c}.0004 \\
(.0002)\end{array}$ & $\begin{array}{c}.0714 \\
(.0026) \\
\end{array}$ \\
\hline & & 2007 & $\begin{array}{c}13.5236 \\
(.1827) \\
\end{array}$ & $\begin{array}{l}13.7103 \\
(.1557) \\
\end{array}$ & $\begin{array}{c}.0740 \\
(.0730) \\
\end{array}$ & $\begin{array}{c}.1127 \\
(.0053) \\
\end{array}$ & $\begin{array}{c}.1875 \\
(.0070) \\
\end{array}$ & $\begin{array}{c}.0005 \\
(.0002) \\
\end{array}$ & $\begin{array}{c}.0744 \\
(.0031) \\
\end{array}$ \\
\hline \multirow{3}{*}{0.70} & \multirow{3}{*}{0.50} & 2004 & $\begin{array}{c}13.3800 \\
(.1737) \\
\end{array}$ & $\begin{array}{l}13.5832 \\
(.1843) \\
\end{array}$ & $\begin{array}{c}.0699 \\
(.0602) \\
\end{array}$ & $\begin{array}{c}.1333 \\
(.0052) \\
\end{array}$ & $\begin{array}{c}.2041 \\
(.0072) \\
\end{array}$ & $\begin{array}{c}.0007 \\
(.0002) \\
\end{array}$ & $\begin{array}{c}.0702 \\
(.0025) \\
\end{array}$ \\
\hline & & 2006 & $\begin{array}{c}12.9243 \\
(.1726)\end{array}$ & $\begin{array}{c}13.0900 \\
(.1327)\end{array}$ & $\begin{array}{c}.0592 \\
(.0593)\end{array}$ & $\begin{array}{c}.1065 \\
(.0040)\end{array}$ & $\begin{array}{c}.1662 \\
(.0057)\end{array}$ & $\begin{array}{c}.0006 \\
(.0004)\end{array}$ & $\begin{array}{c}.0590 \\
(.0021)\end{array}$ \\
\hline & & 2007 & $\begin{array}{c}13.4129 \\
(.1684) \\
\end{array}$ & $\begin{array}{c}13.5977 \\
(.1305) \\
\end{array}$ & $\begin{array}{c}.0620 \\
(.0609) \\
\end{array}$ & $\begin{array}{c}.1228 \\
(.0051) \\
\end{array}$ & $\begin{array}{c}.1883 \\
(.0068) \\
\end{array}$ & $\begin{array}{c}.0011 \\
(.0005) \\
\end{array}$ & $\begin{array}{c}.0644 \\
(.0023) \\
\end{array}$ \\
\hline \multirow{3}{*}{0.70} & \multirow{3}{*}{0.65} & 2004 & $\begin{array}{l}13.4982 \\
(.2072) \\
\end{array}$ & $\begin{array}{c}13.6968 \\
(.1899) \\
\end{array}$ & $\begin{array}{c}.0763 \\
(.0638) \\
\end{array}$ & $\begin{array}{c}.1223 \\
(.0038) \\
\end{array}$ & $\begin{array}{c}.1994 \\
(.0070) \\
\end{array}$ & $\begin{array}{c}.0008 \\
(.0003) \\
\end{array}$ & $\begin{array}{c}.0764 \\
(.0032) \\
\end{array}$ \\
\hline & & 2006 & $\begin{array}{c}13.0280 \\
(.1632) \\
\end{array}$ & $\begin{array}{l}13.1824 \\
(.1419) \\
\end{array}$ & $\begin{array}{c}.0625 \\
(.0648) \\
\end{array}$ & $\begin{array}{c}.0919 \\
(.0038) \\
\end{array}$ & $\begin{array}{c}.1564 \\
(.0052) \\
\end{array}$ & $\begin{array}{c}.0005 \\
(.0002) \\
\end{array}$ & $\begin{array}{c}.0640 \\
(.0024) \\
\end{array}$ \\
\hline & & 2007 & $\begin{array}{c}13.5361 \\
(.1764) \\
\end{array}$ & $\begin{array}{l}13.7172 \\
(.1515) \\
\end{array}$ & $\begin{array}{c}.0674 \\
(.0639) \\
\end{array}$ & $\begin{array}{c}.1136 \\
(.0056) \\
\end{array}$ & $\begin{array}{c}.1808 \\
(.0068) \\
\end{array}$ & $\begin{array}{c}.0008 \\
(.0003) \\
\end{array}$ & $\begin{array}{c}.0664 \\
(.0026) \\
\end{array}$ \\
\hline \multirow{3}{*}{0.70} & \multirow{3}{*}{0.80} & 2004 & $\begin{array}{c}13.1970 \\
(.1989) \\
\end{array}$ & $\begin{array}{c}13.4103 \\
(.1727) \\
\end{array}$ & $\begin{array}{c}.0876 \\
(.0745) \\
\end{array}$ & $\begin{array}{c}.1258 \\
(.0055) \\
\end{array}$ & $\begin{array}{c}.2134 \\
(.0081) \\
\end{array}$ & $\begin{array}{c}.0006 \\
(.0002) \\
\end{array}$ & $\begin{array}{c}.0871 \\
(.0025) \\
\end{array}$ \\
\hline & & 2006 & $\begin{array}{c}12.8276 \\
(.1806) \\
\end{array}$ & $\begin{array}{l}12.9895 \\
(.1525) \\
\end{array}$ & $\begin{array}{c}.0703 \\
(.0652) \\
\end{array}$ & $\begin{array}{c}.0916 \\
(.0042) \\
\end{array}$ & $\begin{array}{c}.1644 \\
(.0061)\end{array}$ & $\begin{array}{c}.0005 \\
(.0002) \\
\end{array}$ & $\begin{array}{c}.0723 \\
(.0025) \\
\end{array}$ \\
\hline & & 2007 & $\begin{array}{l}13.2989 \\
(.1723) \\
\end{array}$ & $\begin{array}{l}13.4902 \\
(.1715) \\
\end{array}$ & $\begin{array}{c}.0757 \\
(.0669) \\
\end{array}$ & $\begin{array}{c}.1156 \\
(.0054) \\
\end{array}$ & $\begin{array}{c}.1933 \\
(.0073) \\
\end{array}$ & $\begin{array}{c}.0006 \\
(.0003) \\
\end{array}$ & $\begin{array}{c}.0771 \\
(.0029) \\
\end{array}$ \\
\hline \multirow{3}{*}{\multicolumn{2}{|c|}{ Individuals }} & 2004 & $\begin{array}{c}14.3699 \\
(.1253)\end{array}$ & $\begin{array}{c}14.5259 \\
(.1111)\end{array}$ & $\begin{array}{c}.0584 \\
(.0307)\end{array}$ & $\begin{array}{c}.0976 \\
(.0035)\end{array}$ & $\begin{array}{c}.1562 \\
(.0053)\end{array}$ & $\begin{array}{c}.0001 \\
(.0000)\end{array}$ & $\begin{array}{c}.0586 \\
(.0020)\end{array}$ \\
\hline & & 2006 & $\begin{array}{c}13.4535 \\
(.1198) \\
\end{array}$ & $\begin{array}{c}13.6482 \\
(.1105) \\
\end{array}$ & $\begin{array}{c}.0676 \\
(.0358) \\
\end{array}$ & $\begin{array}{c}.1272 \\
(.0049) \\
\end{array}$ & $\begin{array}{c}.1926 \\
(.0066) \\
\end{array}$ & $\begin{array}{c}.0001 \\
(.0000) \\
\end{array}$ & $\begin{array}{c}.0654 \\
(.0022) \\
\end{array}$ \\
\hline & & 2007 & $\begin{array}{l}14.0034 \\
(.12615) \\
\end{array}$ & $\begin{array}{l}14.1943 \\
(.0003) \\
\end{array}$ & $\begin{array}{c}.0660 \\
(.0391) \\
\end{array}$ & $\begin{array}{c}.1250 \\
(.0046) \\
\end{array}$ & $\begin{array}{c}.1945 \\
(.0065) \\
\end{array}$ & $\begin{array}{c}.0001 \\
(.0000) \\
\end{array}$ & $\begin{array}{c}.0695 \\
(.0022) \\
\end{array}$ \\
\hline
\end{tabular}

Source: Own elaborations. 
Looking at individual nominal incomes, the maximum is in 2004 and the minimum in 2006 for both indexes: differences between associated $R E$ and $V$ do not exceed $0.2 \%$ of the corresponding pre-tax Gini index. $H$ and $R^{*}$ never exceed, respectively, 0.09 and 0.14 .

However, whereas $H$ presents standard errors which are almost equal to $H$ punctual estimates themselves (sometimes even greater), $R^{*}$ standard errors are twenty times less than $R^{*}$ estimates: it follows that there is a significative group overlapping component which lowers the potential redistributive effect $V$, whilst the within group inequality component $H$ is never significantly different from zero.

$R$ and $R_{3}$ reports very small relative standard errors as well: 30 times lower that their respective estimates; even the negligible $R_{2}$ estimates present rather relatively small standard errors.

When we pass to consider reranking effects of tax systems, looking at household equivalent incomes, both overall reranking $R$ index and interval overlapping $R^{*}$ as well as within interval $R_{3}$ indexes present maximum values in 2004 and minimum values in 2006: 2007 estimates are at an intermediate position. Evaluating tax systems from nominal individual incomes, 2004 has minimum horizontal iniquity indexes; however whilst the group overlapping index $R^{*}$ is maximum in 2006, $R$ and $R_{3}$ register their maximum value in 2007.

The 2007 tax reform has significantly ${ }^{14}$ increased the overall reranking index $R$, the group overlapping index, both with respect nominal individual incomes and family equivalent ones ${ }^{15}$. The within group reranking index $R_{3}$ is always significantly different both between 2004 and 2006-2007 for individual incomes; it is not significantly different from zero just for family equivalent incomes between 2006 and 2007.

Conversely, $R^{*}=R_{1}$ is always significantly different both between 2004 and 20062007 for equivalent family incomes; it is not significantly different from zero for individual incomes just between 2006 and 2007.

When considering households equivalent incomes under the aspect of minimal reranking, the 2006 tax system is the best and the 2004 one is the worst among the three

\footnotetext{
14 Differences between pair of indexes have been tested both (i) assuming normality by the test function $($ Index $1-$ Index 2$) / s e\{$ Index $1-$ Index 2$\}$, having estimated the standard error se $\{$ Index $1-\operatorname{Index} 2\}$ by 200 bootstrap replications and (ii) by checking if zero is included in the $95 \%$ percentile interval estimated for (Index 1 - Index 2 ) by 200 bootstrap replications. ${ }^{15}$ It is rather is amazing that 2007 reform $R$ increases also for individuals with respect to 2006 tax system.
} 
here analyzed: the 2007 system appears as a worsening of the 2006 one, even if it remains still better than the 2004 system. When considering individual nominal incomes, the best oriented appears to be the 2004 system, but, amazingly, the 2007 reform not only is worse than the 2004 system, but it seems to be a bit worse even than the 2006 one $R^{*}=R_{1}$ is the only reranking index which is lower in 2007 than in 2006, but the difference is not significantly different from zero.

We observe that if we consider $V, H, R^{*}$ and $R_{3}$ as percentages of their associated $R E$, when $\frac{V}{R E}$ is maximum, generally $\frac{H}{R E}, \frac{R^{*}}{R E}, \frac{R}{R E}$, and $\frac{R_{3}}{R E}$ are maximum too; the same happens, even with some exceptions, mostly for $R_{3} / R E$, when $V / R E$ is minimum.

The here considered tax systems seems to show a positive relation between redistributive effect and reranking effects. In what it concerns the parameters attributed to the Cutler scale, if we decide to base our choice on the global reranking index $R$, we conclude that the optimal value for $\beta$ seems to be 0.65 , whilst different values of $\alpha$ do not seem to modify substantially reranking indexes.

\section{Conclusions}

When close pre-tax equals groups instead of exact pre-tax ones are considered, the residual component in the original Aronson, Johnson and Lambert (1994) model is not the Atkinson-Plotnick-Kakwani index, but only the transvariation index among groups.

In addition, other two components should be considered when close pre-tax equals are used in empirical applications: the reranking of the mean post-tax income among groups and the reranking within groups. Then a further problem arises: an optimal bandwidth must be chosen in order to properly decompose the redistributive effect into vertical, horizontal and reranking effect.

Van de Ven, Creedy and Lambert (2001) individuate the optimal bandwidth that should be used in decomposing the redistributive effect as the Aronson, Johnson and Lambert (1994) methodology suggests without considering the different contribution of the reranking of the mean post-tax income among groups and the reranking within groups. Following Lambert and Urban (2005), we use a decomposition of the Atkinson- 
Plotnick-Kakwani index in three terms in order to propose a new measurement methodology for the individuation of the optimal bandwidth.

According to our analysis, it should be individuated by looking not only to the highest vertical contribution to the redistributive effect, as van de Ven, Creedy and Lambert (2001) suggest, but also to the horizontal inequity due to the reranking of the mean post-tax income among groups: we chose the optimal bandwidth which can lead to the highest vertical effect in presence of no reranking of the mean post-tax income among groups.

In what it concerns the Bank of Italy survey, it seems that a good bandwidth to perform tax evaluations in Italy could be 700 euro large: $\frac{V}{R E}$ corresponding to this bandwidth, if not an absolute maximum, lies in the set of highest $\frac{V}{R E}$ values, moreover, the average group reranking index assumes negligible values.

On the basis of this methodology, we compare changes in the tax code proposed in Italy during the period 2004-2007. The 2006 tax law shows lower reranking effects if households equivalent incomes are considered, whilst the 2004 tax law presents minimal reranking effects if individuals incomes are considered; 2007 reranking effects lie between 2004 and 2006 ones for equivalent family incomes, whilst they are the highest for individual incomes. Of course family equivalent income analysis depend on the here adopted equivalence scales; in order to limit scale arbitrariness we applied the Cutler scale, allowing for the children parameter $\alpha$ and the overall parameter $\beta$ to assume each three different values: $(0.30 ; 0.50 ; 0.70)$ for the former and $(0.50 ; 0.65$; $0.80)$ for the latter. 


\section{References}

Aronson R. J., Lambert P. J., (1993), "Inequality decomposition analysis and the Gini coefficient revisited", The Economic Journal, 103, pp. 1221-1227.

Aronson R. J., Lambert. P. J., (1994), “Decomposing the Gini coefficient to reveal the vertical, horizontal and reranking effects of income taxation”, National Tax Journal, 47, pp. 273-294.

Aronson R. J., Johnson P. J., Lambert P. J., (1994), "Redistributive effect and unequal income tax treatment", The Economic Journal, 104, pp. 262-270.

Dagum C. (1997), "A new approach to the decomposition of Gini income inequality ratio", Empirical Economics, 22, pp. 515-531.

Ebert U., Moyes P., (2000), “Consistent income tax structures when households are heterogeneous”, Journal of Economic Theory, 90, pp. 116-150.

Lambert P.J., Ramos X., (1997a), "Horizontal inequity and vertical redistribution”, International Tax and Public Finance, 4, pp. 25-37.

Lambert P.J., Ramos X., (1997b), "Horizontal inequity and reranking”, Research on Economic Inequality, 7, pp. 1-18.

Pellegrino S., (2007a), "Struttura ed effetti redistributivi dell'imposta personale e italiana: il confronto 2000-2005 ed un esercizio di modifica", Economia Pubblica, 37, n. 1-2, forthcoming.

Pellegrino S., (2007b), "Il Modello di microsimulazione IRPEF 2004”, SIEP, Italian Society of Public Economics, WP 583/07.

Pellegrino S., (2007c), “IRPEF 2007: una redistribuzione (quasi) irrilevante?", Rivista di Diritto Finanziario e Scienza delle Finanze, forthcoming.

Urban I., Lambert P. J., (2005), "Redistribution, horizontal inequity and reranking: how to measure them properly", University of Oregon, Working paper.

van de Ven J., Creedy J., Lambert P. J., (2001), “Close equals and calculation of the vertical, horizontal and reranking effects of taxation", Oxford Bulletin of Economics and Statistics, 63, pp. 381-394.

Vernizzi A., (2006), “Una precisazione sulla scomposizione dell'indice di redistribuzione RE di AronsonJohnson-Lambert e una proposta di estensione dell'indice di Plotnick", Economia Pubblica, 37, n. 12, forthcoming, and DEAS, Università degli Studi di Milano, WP 28/06. 